\title{
The Nonlinear Stability Prediction and FEM Modeling of High-Speed Spindle System with Joints Dynamic Characteristics
}

\author{
Bo Wang, ${ }^{1}$ Wei Sun, ${ }^{1}$ Kunpeng Xu, ${ }^{2}$ Junyong Zhang, ${ }^{3}$ and Bangchun Wen ${ }^{1}$ \\ ${ }^{1}$ School of Mechanical Engineering \& Automation, Northeastern University, Shenyang 110819, China \\ ${ }^{2}$ SANY Group Co., Ltd., Shenyang 110027, China \\ ${ }^{3}$ No. 93115 of the People's Liberation Army, Shenyang 110031, China \\ Correspondence should be addressed to Bo Wang; wangb@me.neu.edu.cn
}

Received 14 November 2013; Accepted 5 March 2014; Published 20 May 2014

Academic Editor: Valder Steffen Jr.

Copyright (c) 2014 Bo Wang et al. This is an open access article distributed under the Creative Commons Attribution License, which permits unrestricted use, distribution, and reproduction in any medium, provided the original work is properly cited.

\begin{abstract}
When predicting the nonlinear stability of high-speed spindle system, it is necessary to create an accurate model that reflects the dynamic characteristics of the whole system, including the spindle-bearing joint and spindle-holder-tool joints. In this paper, the distribution spring model of spindle-holder-tool joints was built with the consideration of its dynamic characteristics; the five-DOF dynamic model of the angle contact ball bearing was also established to study the influence of speed and preload on the spindlebearing joint, both of which were used in the general whole complete spindle system FEM model. The rationality of the model was verified by comparison with the FRF of traditional rigid model and experiments. At last, the influences of speed and cutting force on the nonlinear stability were analyzed by amplitude spectrum, bifurcation, and Poincaré mapping. The results provided a theoretical basis and an evaluating criterion for nonlinear stability prediction and product surface quality improvement
\end{abstract}

\section{Introduction}

High-speed machining technology is widely used in mould, aircraft structural components, and some other volume productions industry for productivity rising and costs reducing which will lead to poor surface finish, reduced material removal rate, high noise, and tool or work piece damage simultaneously. With the processing speed becoming higher and higher, the cutting stability affected parameters are different from traditional ones, especially the structure of high-speed spindle system. The traditional spindle is rigidity which works under the first-order critical speed; however, the processing speed is getting higher at present, which not only causes the centrifugal force, the gyroscopic moment, and speed-dependent bearing stiffness to be nonignored, but also makes the joints influence amplified.

The construction of high-speed spindle system becomes a critical factor to affect the dynamics and the cutting stability, especially the spindle joints including spindle-bearing joint and spindle-holder-tool joints. The structure of spindlebearing joint is familiar and its dynamic characteristics are decided by the bearing. The spindle-holder-tool joints consist of two parts: the spindle-holder joint is a conical surface which is the part of holder inserting spindle; the holder-tool joint is a cylindrical surface which is the part of tool inserting holder.

The research of spindle-bearing joint has been done for decades and some achievements have been created. Cao and Altintas [1] have modeled the spindle-bearing with the Timoshenko using the Jones bearing model in form of force. Shin [2] has presented $6 \times 6$ bearing stiffness matrix with Jacobian matrix. Chen et al. [3] have built nonlinear dynamic model of rotor system with rolling bearing.

Spindle-holder-tool joints are other significant factors in influencing the high-speed spindle system dynamic characteristics besides spindle-bearing joint. Rivin [4] has proposed the six important aspects of machining tool research, in which the joint structure and dynamic related study will become of emphasis in the future. Schmitz et al. [5] have modeled and analyzed the spindle joints considering translation degree. Namazi et al. [6] have built the contact stiffness and damping model to simulate the joint surface loose by moving the spring element. Ertürk et al. [7] have assumed the radial contact between spindle and holder ignoring 
the taper joint surface between holder and tool. Yumei et al. [8] have taken the joint between spindle and holder of JCS-018 as example to research dynamic characteristics of fixed taper surface. Zhang et al. [9] have discussed the stress distribution between contact surfaces.

The final objective of spindle joints modeling and analysis is to predict the stability of machining center. On macroscopic view, the methods of cutting stability research will be divided into two ways: online monitoring and offline predicting. Winfough [10] has developed the online method for detecting the chatter phenomenon during the process with microphones. The result of online method could only be used to confirm the stability of that process; besides, the external environment is so changeable from moment to moment that weakens the reference for other cutting processes. Therefore, the offline method with lobe diagrams has been proposed to predict the cutting process stability since the 1960s by Koenigsberger and Tlusty [11], Tobias [12], and Merrit [13]. Park et al. [14, 15] have developed stability lobe diagrams for prediction with analytical utility. Mann et al. [16] have proposed a new method to obtain stability lobe diagrams in upmilling and downmilling machining. Schmitz et al. [17] have confirmed that the maximum cutting depth on static is lower than the actual. Weixiao [18] has established the dynamic cutting force model to research the stability criterion.

The stability prediction method develops from traditional two-dimensional lobes to three-dimensional lobes for improving the prediction accuracy with the increased product quality requirement and complicated working condition. Tang and Liu [19] have gotten the three-dimensional lobes of thin-walled workpiece. Abrari et al. [20] have drawn the limit stability diagram of spherical cutter at different milling position. Dai Depai [21] has proposed an equivalent linearization method to obtain a three-dimensional stability chart. Qinghua [22] has structured three- dimensional limit chart for thin-walled workpiece milling system.

In this study, the FEM of high-speed spindle system was built with joints characteristics considering the high speed influence. The spindle-bearing joint was structured with angle contact ball bearing dynamic model affected by speed and preload; the spindle-holder-tool joints were simulated in distributed spring with hysteretic nonlinearity. The model was more rational than traditional rigid connection verified by experiment. Based on this, the influence of speed and cutting force at tool point on processing stability was analyzed. The nonlinear response and stability of tool point were evaluated by amplitude spectrum and bifurcation and Poincaré mapping which could provide a reliable basis for enhancing the nonlinear stability and improving the machining accuracy.

\section{Modeling}

The high-speed spindle system dynamic model is the foundation and premise of dynamic response and cutting stability, which includes three parts: spindle-bearing modeling,

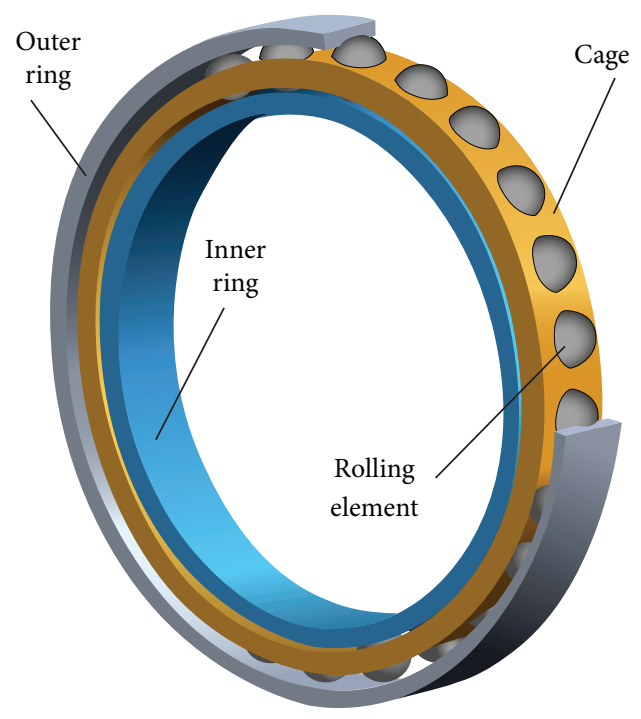

FIGURE 1: The structure of ceramic ball bearing.

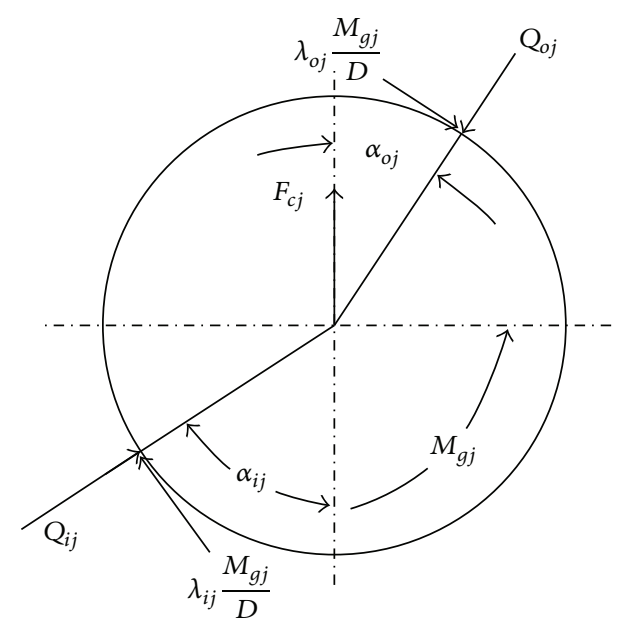

Figure 2: The forces on the roller.

spindle-holder-tool joints modeling, and the complete spindle system model with all the above.

2.1. Bearing Joint Modeling. At present, the hybrid ceramic ball bearing is used to satisfy the requirement of stiffness and rotational speed in high-speed spindle widely, as shown in Figure 1. The dynamic loading between the rolling element and raceway is produced because of the rolling revolution and rotation, the centrifugal force, and the gyroscopic moment, the distribution of which will be changed under the high speed condition. The displacement and load properties of bearing even the dynamic characteristics of spindle system with the bearing will be affected when the dynamic loading reaches a significant level.

The force distribution of one rolling element is shown in Figure 2, which will make pure rolling according to the raceway control theory when the speed increases; then the gyroscopic moment of ball is balanced with friction of outer 


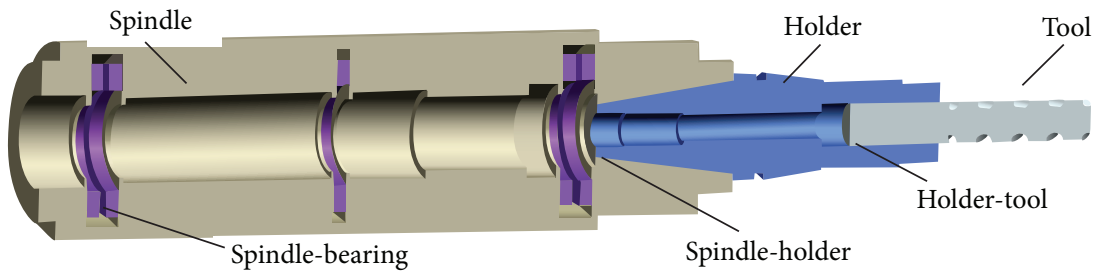

FIgURE 3: The cutaway view of spindle system.

ring; that is, $\lambda_{i j}=0, \lambda_{o j}=2$. Each rolling element is a balance system; that is,

$$
\begin{gathered}
Q_{i j} \sin \alpha_{i j}-Q_{o j} \sin \alpha_{o j}+\frac{2 M_{g j}}{D} \cos \alpha_{o j}=0, \\
Q_{i j} \cos \alpha_{i j}-Q_{o j} \cos \alpha_{o j}+\frac{2 M_{g j}}{D} \sin \alpha_{o j}+F_{c j}=0,
\end{gathered}
$$

where $Q_{i j}, Q_{o j}$ are normal contact load between rolling element and inner or outer ring, $Q_{n j}=K_{n j} \delta_{n j}^{1.5}(n=$ $i, o), \alpha_{i j}, \alpha_{o j}$ are contact angle between rolling element and inner or outer ring, $F_{c j}$ is centrifugal force of rolling element, $M_{g j}$ is gyroscopic moment of rolling element, and $D$ is rolling element diameter.

The forces between spindle unit and inner ring of bearing are axial force $F_{x}$, radial forces $F_{y}$ and $F_{z}$, and torque $M_{y}$ and $M_{z}$; even considering the forces between rolling element and inner ring of bearing, the equilibrium equation of inner ring is

$$
\begin{gathered}
F_{x}-\sum_{j=1}^{N_{b}} Q_{i j} \sin \alpha_{i j}=0, \\
F_{y}-\sum_{j=1}^{N_{b}} Q_{i j} \cos \alpha_{i j} \cos \psi_{j}=0, \\
F_{z}-\sum_{j=1}^{N_{b}} Q_{i j} \cos \alpha_{i j} \sin \psi_{j}=0, \\
M_{y}-\sum_{j=1}^{N_{b}} Q_{i j} \cos \alpha_{i j} \Re_{i} \sin \psi_{j}=0, \\
M_{z}-\sum_{j=1}^{N_{b}} Q_{i j} \sin \alpha_{i j} \Re_{i} \cos \psi_{j}=0,
\end{gathered}
$$

where $\mathfrak{R}_{i}$ is radius of inner ring curvature center and $\psi_{j}$ is position angle.

With the high speed processing condition, the deformation equation of one supported bearing in matrix form is

$$
\left\{\begin{array}{c}
F_{x} \\
F_{y} \\
F_{z} \\
M_{y} \\
M_{z}
\end{array}\right\}=\left(\begin{array}{lllll}
k_{\delta_{x} \delta_{x}} & k_{\delta_{x} \delta_{y}} & k_{\delta_{x} \delta_{z}} & k_{\delta_{x} \theta_{y}} & k_{\delta_{x} \theta_{z}} \\
k_{\delta_{y} \delta_{x}} & k_{\delta_{y} \delta_{y}} & k_{\delta_{y} \delta_{z}} & k_{\delta_{y} \theta_{y}} & k_{\delta_{y} \theta_{z}} \\
k_{\delta_{z} \delta_{x}} & k_{\delta_{z} \delta_{y}} & k_{\delta_{z} \delta_{z}} & k_{\delta_{z} \theta_{y}} & k_{\delta_{z} \theta_{z}} \\
k_{\theta_{y} \delta_{x}} & k_{\theta_{y} \delta_{y}} & k_{\theta_{y} \delta_{z}} & k_{\theta_{y} \theta_{y}} & k_{\theta_{y} \theta_{z}} \\
k_{\theta_{z} \delta_{x}} & k_{\theta_{z} \delta_{y}} & k_{\theta_{z} \delta_{z}} & k_{\theta_{z} \theta_{y}} & k_{\theta_{z} \theta_{z}}
\end{array}\right)\left\{\begin{array}{l}
\delta_{x} \\
\delta_{y} \\
\delta_{z} \\
\theta_{y} \\
\theta_{z}
\end{array}\right\}
$$

simplified as

$$
\mathbf{F}_{b}=\mathbf{K}_{b} \boldsymbol{\delta}_{b}
$$

where $\mathbf{F}_{b}$ is the spindle-bearing force vector, $\mathbf{K}_{b}$ is bearing dynamic stiffness matrix, and $\boldsymbol{\delta}_{b}$ is bearing deformation vector.

The relationship between load and deformation of fiveDOF ball bearing is listed in (3), which could be used in dynamics equation of system to realize the whole spindlebearing system model in load-deformation way or could get stiffness matrix $\mathbf{K}_{b}$ used in the FEM model directly.

2.2. Spindle-Holder-Tool Joints Modeling. In this section, the spindle-holder-tool joints model will be built with FEM and the dynamic characteristics will be simulated with stiffness and damping matrices. The structure of spindle-holder-tool is double parallel rotor system which consists of two parts: one parallel rotor is spindle and holder; the other one is holder and tool shown in Figure 3.

The nodes are numbered after the rotor system becomes discrete; then a transition section with random information is added at the end of outer rotor and the inner rotor numbers will be continuous. The mass and stiffness matrices of the whole system are assembled with the ones of each unit excluding the transition section.

Assuming $p$ units in outer rotor and $q$ units in inner rotor, there are $p+q+1$ units in the parallel rotor system and $N$ nodes, $N=p+1+1+1+q+1$. The mass and stiffness matrices of each unit are $12 \times 12$ orders, which consist of two nodes and each node owns six degrees of freedom. The assembly of adjacent units is shown in Figure 4. The mass and stiffness matrices of the whole system are $6 \mathrm{~N} \times 6 \mathrm{~N}$ orders, from which the transition section matrices will be removed. The final complete mass and stiffness matrices of parallel rotor are shown in Figure 5.

The simulated method of joint dynamic characteristics is illustrated by taking the spindle-holder joint as an example. The model of spindle and holder joint simulated by distribution spring is shown in Figure 6.

Assuming the spindle and holder joint is divided into $n$ stepped shafts, in the joint there are $n+1$ nodes, $i, i+1, \ldots, i+n$, on the spindle and $j, j+1, \ldots j+n$ on the holder, respectively. The coupling forces on the spindle and holder are

$$
\begin{aligned}
\Delta \mathbf{F}_{i} & =\mathbf{K}_{\text {coup }} \mathbf{X}_{i}+\mathbf{C}_{\text {coup }} \dot{\mathbf{X}}_{i}-\mathbf{K}_{\text {coup }} \mathbf{X}_{j}-\mathbf{C}_{\text {coup }} \dot{\mathbf{X}}_{j} \\
\Delta \mathbf{F}_{j} & =\mathbf{K}_{\text {coup }} \mathbf{X}_{j}+\mathbf{C}_{\text {coup }} \dot{\mathbf{X}}_{j}-\mathbf{K}_{\text {coup }} \mathbf{X}_{i}-\mathbf{C}_{\text {coup }} \dot{\mathbf{X}}_{i}
\end{aligned}
$$




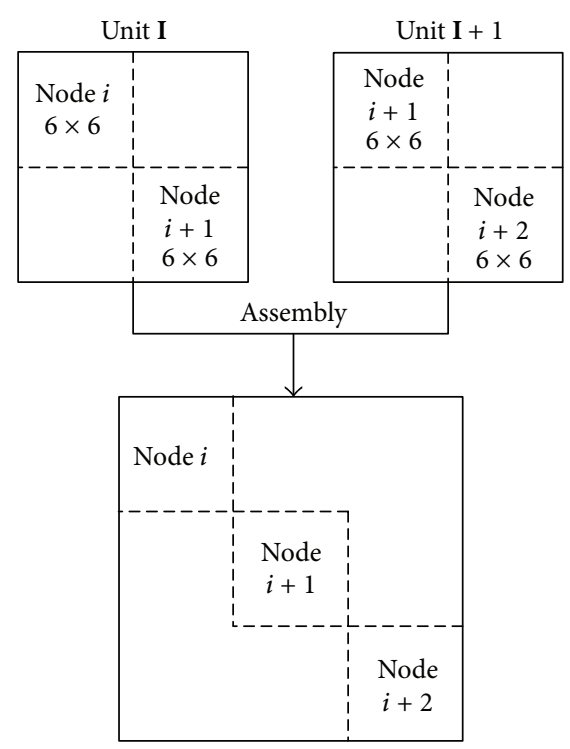

FIgURE 4: Schemes of adjacent unit assembly.

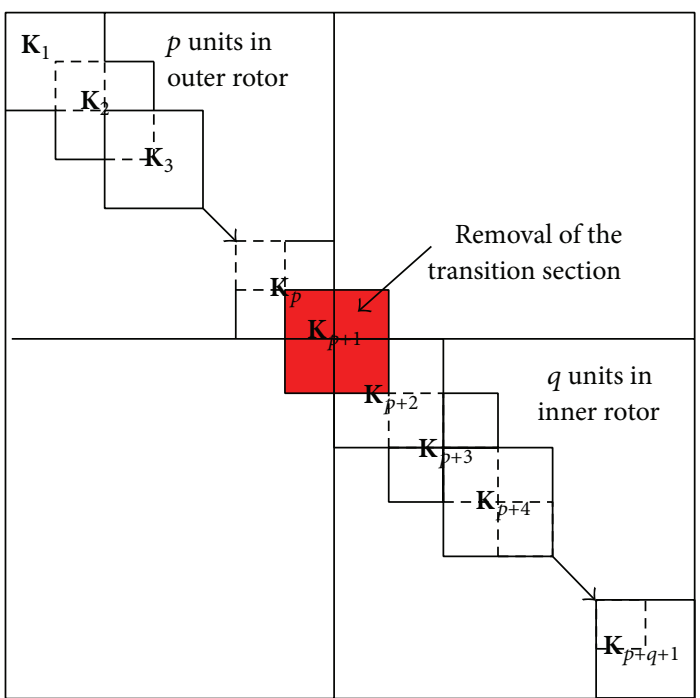

FIGURE 5: Schemes of parallel rotors assembly.

$\Delta \mathbf{F}_{i+1}=\mathbf{K}_{\text {coup }} \mathbf{X}_{i+1}+\mathbf{C}_{\text {coup }} \dot{\mathbf{X}}_{i+1}-\mathbf{K}_{\text {coup }} \mathbf{X}_{j+1}-\mathbf{C}_{\text {coup }} \dot{\mathbf{X}}_{j+1}$

$\Delta \mathbf{F}_{j+1}=\mathbf{K}_{\text {coup }} \mathbf{X}_{j+1}+\mathbf{C}_{\text {coup }} \dot{\mathbf{X}}_{j+1}-\mathbf{K}_{\text {coup }} \mathbf{X}_{i+1}-\mathbf{C}_{\text {coup }} \dot{\mathbf{X}}_{i+1}$

$\Delta \mathbf{F}_{i+n}=\mathbf{K}_{\text {coup }} \mathbf{X}_{i+n}+\mathbf{C}_{\text {coup }} \dot{\mathbf{X}}_{i+n}-\mathbf{K}_{\text {coup }} \mathbf{X}_{j+n}-\mathbf{C}_{\text {coup }} \dot{\mathbf{X}}_{j+n}$

$\Delta \mathbf{F}_{j+n}=\mathbf{K}_{\text {coup }} \mathbf{X}_{j+n}+\mathbf{C}_{\text {coup }} \dot{\mathbf{X}}_{j+n}-\mathbf{K}_{\text {coup }} \mathbf{X}_{i+n}-\mathbf{C}_{\text {coup }} \dot{\mathbf{X}}_{i+n}$, where $\mathbf{K}_{\text {coup }}$ and $\mathbf{C}_{\text {coup }}$ are coupling stiffness and damping matrices between spindle and holder.

The stiffness and damping matrices of spindle-holder joint expressed with $\mathbf{K}_{c}$ and $\mathbf{C}_{c}$ are

$$
\begin{aligned}
& \mathbf{K}_{c}=\stackrel{i}{j}\left[\begin{array}{ccc}
\mathbf{K}_{\text {coup }} & & -\mathbf{K}_{\text {coup }} \\
-\mathbf{K}_{\text {coup }} & & \mathbf{K}_{\text {coup }}
\end{array}\right], \\
& \begin{array}{lll}
i & \ldots & j
\end{array} \\
& \mathrm{C}_{c}=\stackrel{i}{j}\left[\begin{array}{ccc}
\mathrm{C}_{\text {coup }} & & -\mathrm{C}_{\text {coup }} \\
& \ddots & \\
-\mathrm{C}_{\text {coup }} & & \mathrm{C}_{\text {coup }}
\end{array}\right] . \\
& \begin{array}{lll}
i & \ldots & j
\end{array}
\end{aligned}
$$

The dynamic characteristics of distribution spring are shown in (8) and the assembly method of stiffness on joint is shown in Figure 7:

$$
\begin{aligned}
\mathbf{K}_{\text {coup }} & =\left[\begin{array}{ccccccc}
0 & 0 & 0 & 0 & 0 & 0 \\
0 & k_{y y} & k_{y z} & 0 & 0 & 0 \\
0 & k_{z y} & k_{z z} & 0 & 0 & 0 \\
0 & 0 & 0 & k_{\theta} & 0 & 0 \\
0 & 0 & 0 & 0 & 0 & 0 \\
0 & 0 & 0 & 0 & 0 & 0
\end{array}\right], \\
\mathbf{C}_{\text {coup }} & =\left[\begin{array}{cccccc}
0 & 0 & 0 & 0 & 0 & 0 \\
0 & c_{y y} & c_{y z} & 0 & 0 & 0 \\
0 & c_{z y} & c_{z z} & 0 & 0 & 0 \\
0 & 0 & 0 & c_{\theta} & 0 & 0 \\
0 & 0 & 0 & 0 & 0 & 0 \\
0 & 0 & 0 & 0 & 0 & 0
\end{array}\right] .
\end{aligned}
$$

2.3. High-Speed Spindle Modeling. The spindle system consists of HCS150 motorized spindle from GMN, HSK-E50 holder, and R245 milling cutter. The FEM model of highspeed spindle system is discretized with different inner or outer diameters, bearing locations, spindle-holder taper joint, and holder-tool cylindrical joint shown in Figure 8. The bolts and lubrication are neglected. The five supporting bearings are installed in two pairs and the middle is independent.

The dots in Figure 9 represent mass point; the small sections represent unit. There are 52 units and 53 nodes in the FEM including 2 transition sections. The external forces on the right consist of spindle-bearing forces, spindle-holdertool joints hysteretic forces, and cutting force on the tool point. The dynamic equation of high-speed spindle system could be expressed as

$$
\begin{gathered}
\mathbf{M} \ddot{\mathbf{X}}+\mathbf{C} \dot{\mathbf{X}}+\mathbf{K} \mathbf{X}=\mathbf{R}(\mathbf{X})+\mathbf{H}(\mathbf{X}, \dot{\mathbf{X}})+\mathbf{P}(t)+\mathbf{G}, \\
\mathbf{C}=\mathbf{D}+\Omega \cdot \mathbf{J}+\mathbf{K}_{H}, \quad \mathbf{K}=\mathbf{K}_{s}+\mathbf{K}_{C} \\
\mathbf{X}=\left[q_{1}, q_{2}, \ldots, q_{n}\right]^{T}, \quad(n=51) \\
q_{i}=\left[x_{i}, y_{i}, z_{i}, \theta_{x i}, \theta_{y i}, \theta_{z i}\right]^{T}, \quad(i=1,2, \ldots, 51),
\end{gathered}
$$




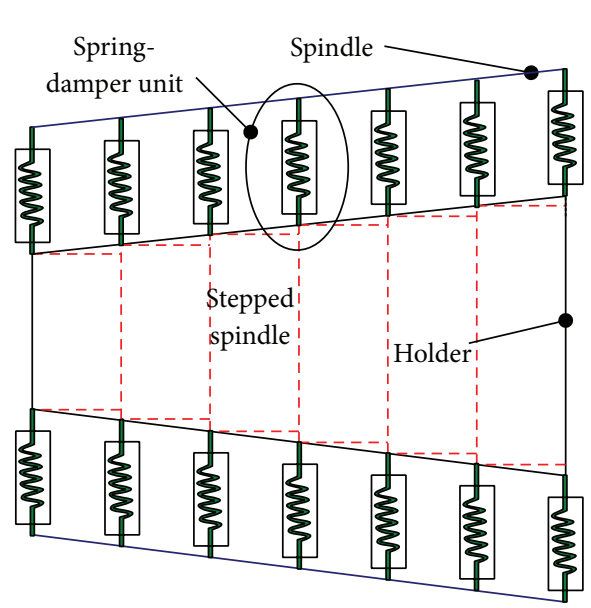

(a) Structure

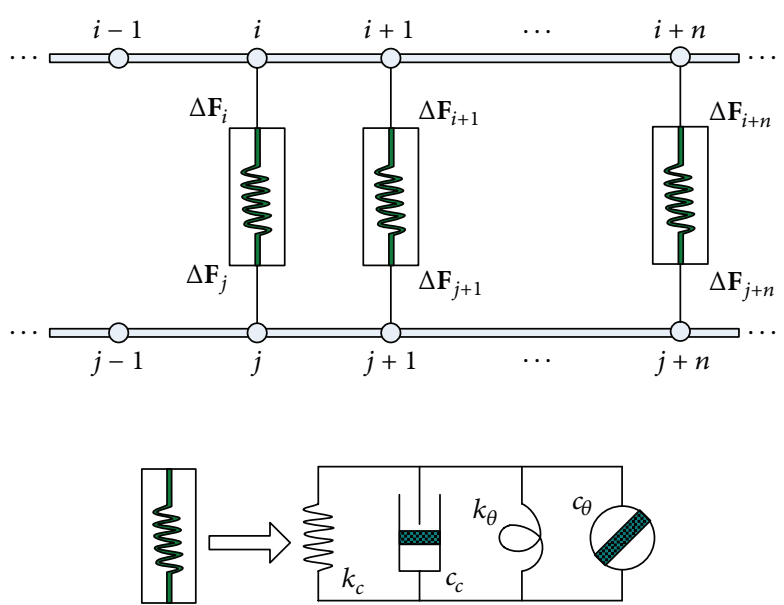

(b) FEM

FIgURE 6: The distribution spring model of spindle-holder joint.

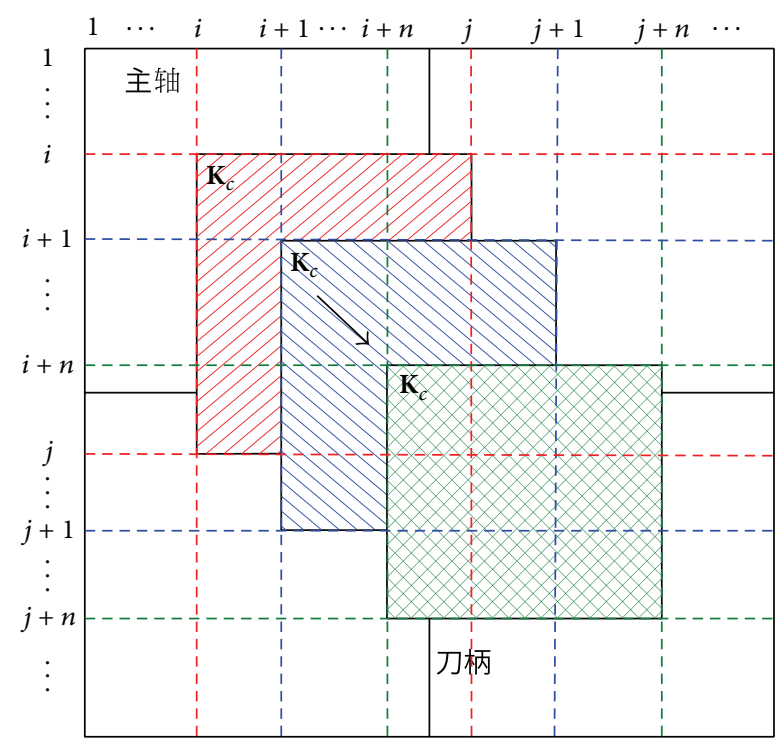

FIGURE 7: Schematic diagram of joint stiffness assembly.

where $\mathbf{M}$ is mass matrix; $\mathbf{C}$ is damping matrix; $\mathbf{K}$ is stiffness matrix; D is damping matrix; $\mathbf{J}$ is gyroscopic matrix; $\mathbf{K}_{H}$ is coupling stiffness matrix of joints; $\mathbf{K}_{s}$ is stiffness matrix of spindle; $\mathbf{K}_{C}$ is centrifugal stiffness matrix of spindle; $\mathbf{R}$ is supporting bearing force vector; $\mathbf{H}$ is hysteretic force vector of spindle-holder-tool; $\mathbf{P}$ is cutting force vector; $\mathbf{G}$ is gravity vector; and $\mathbf{X}$ is displacement vector.

\section{Model Validation}

The modal experiment is done on VMC0540d by hammering to prove the effectiveness of modeling method for spindleholder-tool joints and the whole spindle system. The results obtained from the experiment, distribution spring model,
TABLE 1: The instruments used in this test.

\begin{tabular}{lc}
\hline Number & Name \\
\hline 1 & B\&K 3560-C data acquisition front-end \\
2 & PCB 8206-001 54627 modal hammer \\
3 & B\&K 4525B-001 54072 acceleration sensor \\
4 & High-performance notebook computers \\
\hline
\end{tabular}

and rigid model will be compared by the frequency response function of tool point.

The test system is set up and shown in Figure 9. The main test equipment includes the data acquisition, hammer, and vibration sensors, shown in Table 1.

The FEM of test object is built with distribution spring connection and rigid connection, respectively. The dynamic parameters of system are elasticity modulus $E=2.06 \times$ $10^{11} \mathrm{~Pa}$, material density $\rho=7850 \mathrm{~kg} / \mathrm{m}^{3}$, contact stiffness of spindle-holder-tool joints $k_{s h}=k_{h t}=1 \times 10^{8} \mathrm{~N} / \mathrm{m}, 7011 \mathrm{AC}$ angle contact ball bearing, clearance $r_{0}=1 \mu \mathrm{m}$, and ball stiffness $C_{b}=1 \times 10^{9} \mathrm{~N} / \mathrm{m}^{1.5}$. The structures of spindle system are shown in Tables 2 and 3.

The FRF curves of tool point are drawn in Figure 10 and the results of the first five-order inherence frequencies are listed in Table 4 from experiment and two connection models. The experimental results are affected experimental environment, changing temperature, equipment precision, clamped situation, and some other factors, which lead to the differences between the simulations.

The results got from the distribution spring model are much closer to the experiment than the rigid connection whose error is less than $8 \%$; however, the results from the rigid model even miss the first-order inherence frequency. Therefore, the distribution spring model of spindle-holdertool joints reflects the real connection and dynamic characteristics among spindle, holder, and tool better which should be used in joints and spindle system dynamic modeling. 


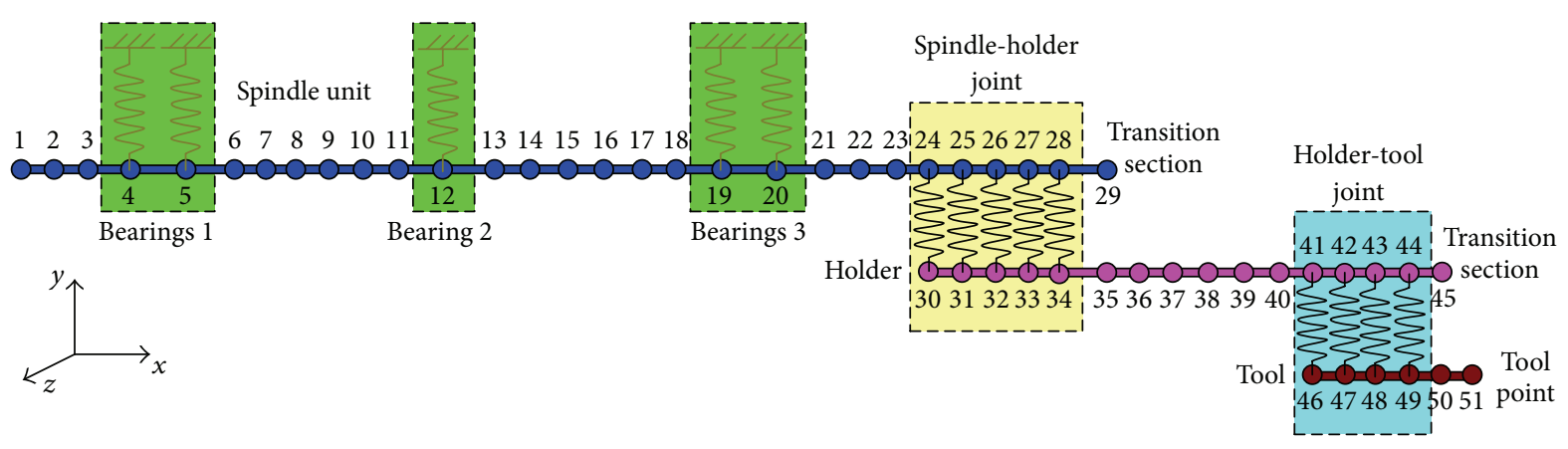

FIGURE 8: The model of high-speed spindle system.

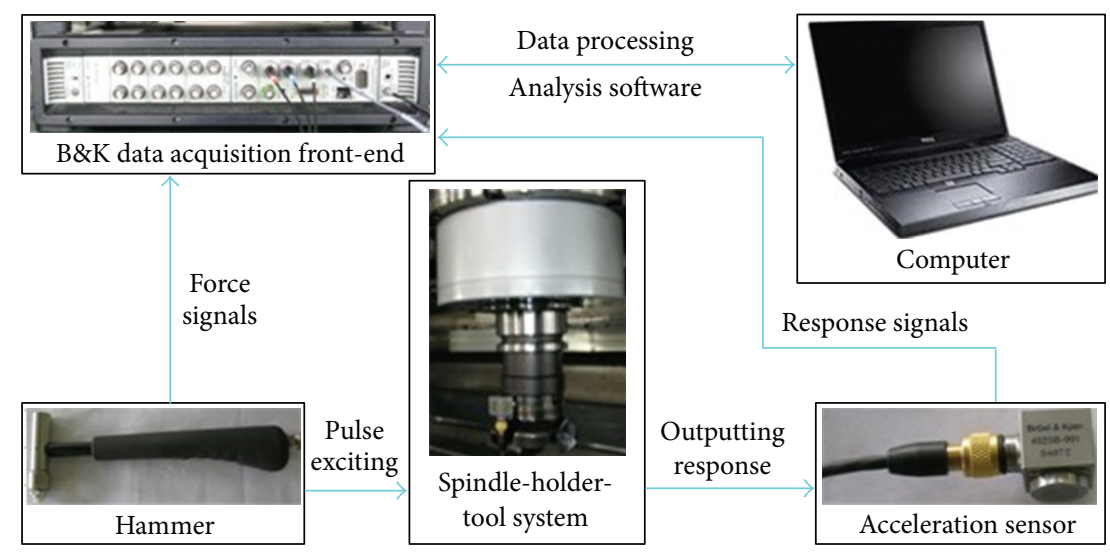

FIgURE 9: The test system.

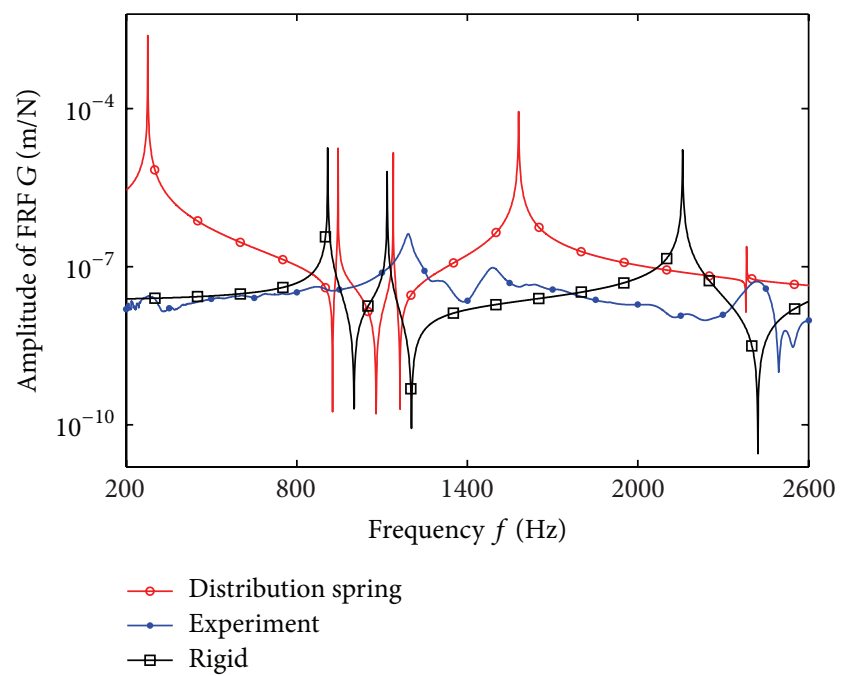

FIGURE 10: The comparison of FRF at tool point.

\section{Model Applications}

The stability is one of the most significant parameters for evaluating characteristics of spindle system and processing quality which could not be predicted accurately by traditional limit cutting depth method with the nonlinear phenomenon remarkable and speed higher especially. On the basis of the spindle system model with distribution spring connection, the effect rules of response and stability at tool point are studied on speed and cutting force amplitude with the bifurcation diagram and Poincare mapping. For the cutting process, the periodic motion is named stability state, and other motions are named instability states including quasiperiodic motion and chaotic motion.

4.1. The Influence of Speed on Stability. The bifurcation diagram at tool point of high-speed spindle system in $y$ direction from $1000 \mathrm{r} / \mathrm{min}$ to $25000 \mathrm{r} / \mathrm{min}$ is shown in Figure 11. The movement state of tool point is chaos in the beginning when the speed is round $1000 \mathrm{r} / \mathrm{min}$, then becomes quasiperiodic motion gradually as the speed increases to $7000 \mathrm{r} / \mathrm{min}$, and finally tends to be stable periodic motion similarly with the speed continuing to rise up. The amplitude spectrum and Poincaré mapping at tool point are shown in Figure 12 to research the stability changing laws with speed including $1200 \mathrm{r} / \mathrm{min}, 7800 \mathrm{r} / \mathrm{min}, 12600 \mathrm{r} / \mathrm{min}$, and $20000 \mathrm{r} / \mathrm{min}$ clearly.

The amplitude spectrum and mapping of tool point at $1000 \mathrm{r} / \mathrm{min}$ are shown in Figures 12(a1)-12(b1). The rotating frequency is $16.67 \mathrm{~Hz}$. There are lots of combination frequencies of inherence frequency and rotating frequency. The system is not stable because there is a similar frequency of $286.1 \mathrm{~Hz}$ near the first-order inherence frequency which is proved by the random points in Poincaré mapping. 
TABLE 2: The size of motorized spindle in $\mathrm{mm}$.

\begin{tabular}{|c|c|c|c|}
\hline Number & $L$ & $D$ & $d$ \\
\hline 1 & 14.5 & 120 & 70 \\
\hline 2 & 25 & 149.9 & 70 \\
\hline 3 & 15 & 149.9 & 55 \\
\hline 4 & 15 & 149.9 & 55 \\
\hline 5 & 25 & 149.9 & 65 \\
\hline 6 & 30 & 149.9 & 65 \\
\hline 7 & 25 & 149.9 & 65 \\
\hline 8 & 25 & 149.9 & 65 \\
\hline 9 & 30 & 150 & 65 \\
\hline 10 & 30 & 150 & 65 \\
\hline 11 & 15 & 150 & 55 \\
\hline 12 & 30 & 150 & 65 \\
\hline 13 & 30 & 150 & 65 \\
\hline 14 & 30 & 150 & 55 \\
\hline 15 & 25 & 150 & 55 \\
\hline 16 & 25 & 150 & 55 \\
\hline 17 & 25 & 150 & 70 \\
\hline 18 & 15 & 150 & 55 \\
\hline 19 & 15 & 150 & 55 \\
\hline 20 & 18 & 149.9 & 55 \\
\hline 21 & 20 & 129.9 & 55 \\
\hline 22 & 20 & 129.9 & 55 \\
\hline 23 & 20 & 129.9 & 55 \\
\hline 24 & 5 & 129.9 & 34 \\
\hline 25 & 5 & 129.9 & 35 \\
\hline 26 & 5 & 129.9 & 36 \\
\hline 27 & 5 & 88 & 37 \\
\hline
\end{tabular}

The amplitude spectrum and Poincaré mapping of tool point at $2000 \mathrm{r} / \mathrm{min}$ are shown in Figures 12(a2)-12(b2). The rotating frequency is $32.71 \mathrm{~Hz}$. The system is a quasiperiodic motion by lots of combination frequencies of inherence frequency and rotating frequency in the amplitude spectrum and a closed pattern in Poincaré mapping.

The amplitude spectrum and Poincaré mapping of tool point at $7800 \mathrm{r} / \mathrm{min}$ are shown in Figures 12(a3)-12(b3). The rotating frequency is $127.9 \mathrm{~Hz}$. The frequencies in the amplitude spectrum are combination of rotating frequency and $15 \mathrm{~Hz}$, while $293 \mathrm{~Hz}$ is the combination of inherent frequency and $15 \mathrm{~Hz}$; therefore, the shape in Poincare mapping is a regular pattern but not closed completely which leads to a transition form between quasiperiodic and chaotic motions.

The amplitude spectrum and Poincaré mapping of tool point at $12600 \mathrm{r} / \mathrm{min}$ are shown in Figures 12(a4)-12(b4). The rotating frequency and one-third frequency are shown in the amplitude spectrum. There are three separate points in the Poincaré mapping which shows that the system performs P-3 periodic motion at this speed moment.

The amplitude spectrum and Poincaré mapping of tool point at $20000 \mathrm{r} / \mathrm{min}$ are shown in Figures 12(a5)-12(b5). There is only the rotating frequency $325.5 \mathrm{~Hz}$ in the amplitude
TABLE 3: The size of holder and tool in $\mathrm{mm}$.

\begin{tabular}{|c|c|c|c|}
\hline Number & $L$ & $D$ & $d$ \\
\hline \multicolumn{4}{|c|}{ Holder } \\
\hline 1 & 5 & 34 & 27 \\
\hline 2 & 5 & 35 & 27 \\
\hline 3 & 5 & 36 & 32 \\
\hline 4 & 5 & 37 & 32 \\
\hline 5 & 5 & 38 & 29 \\
\hline 6 & 8 & 50 & 27 \\
\hline 7 & 8 & 50 & 10 \\
\hline 8 & 4 & 44 & 10 \\
\hline 9 & 6 & 50 & 12 \\
\hline 10 & 16 & 39 & 5 \\
\hline 11 & 13 & 42 & 10 \\
\hline 12 & 5 & 22 & 10 \\
\hline 13 & 6 & 22.1 & 10 \\
\hline 14 & 7 & 22.1 & 10 \\
\hline \multicolumn{4}{|c|}{ Tool } \\
\hline 1 & 5 & 40 & 22 \\
\hline 2 & 6 & 40 & 22.1 \\
\hline 3 & 7 & 42 & 22.1 \\
\hline 4 & 10 & 45 & 25 \\
\hline 5 & 12 & 48 & 25 \\
\hline
\end{tabular}

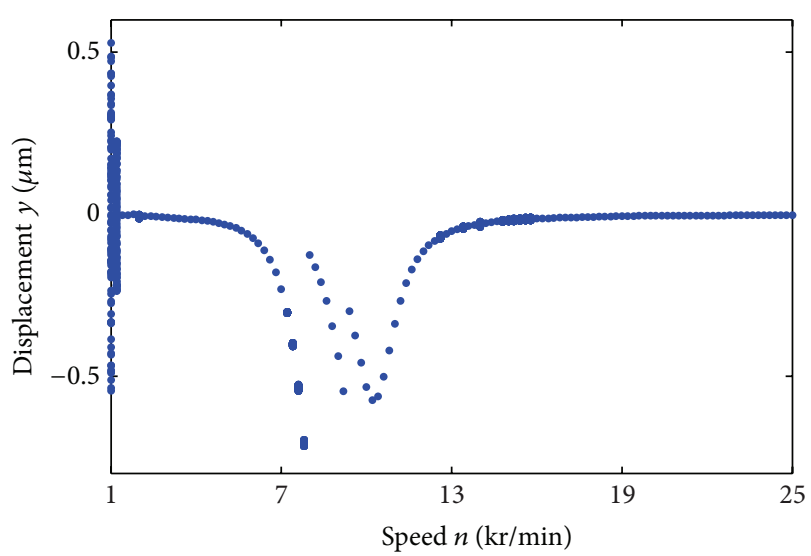

FIgURE 11: Global bifurcation of tool point in $y$ direction with different speeds.

spectrum and one point in Poincaré mapping. The system motion is stable.

4.2. The Influence of Cutting Force on Stability. The bifurcation diagram at tool point of high-speed spindle system in $y$ direction from $10 \mathrm{~N}$ to $120 \mathrm{~N}$ is shown in Figure 13. The displacement at tool point increases with the cutting force obviously, but the stability is ruleless. The system is in quasiperiodic and chaotic states when the cutting force is lower than $50 \mathrm{~N}$, in quasiperiodic and periodic states when the cutting force is between $50 \mathrm{~N}$ and $70 \mathrm{~N}$, and back to the chaotic state again when the force is between $70 \mathrm{~N}$ and $80 \mathrm{~N}$, and when the force continues to rise up, the motion finally 


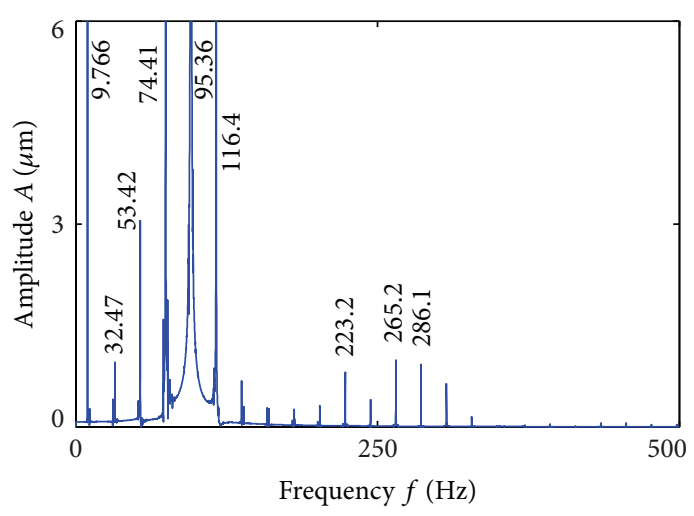

(a1) $1000 \mathrm{r} / \mathrm{min}$ amplitude spectrum

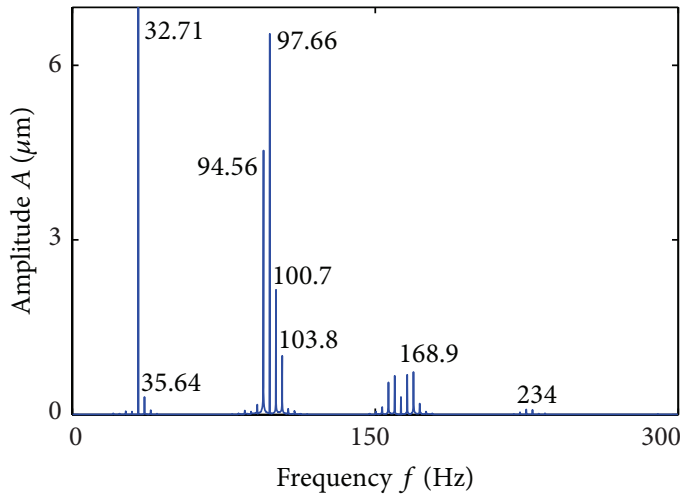

(a2) $2000 \mathrm{r} / \mathrm{min}$ amplitude spectrum

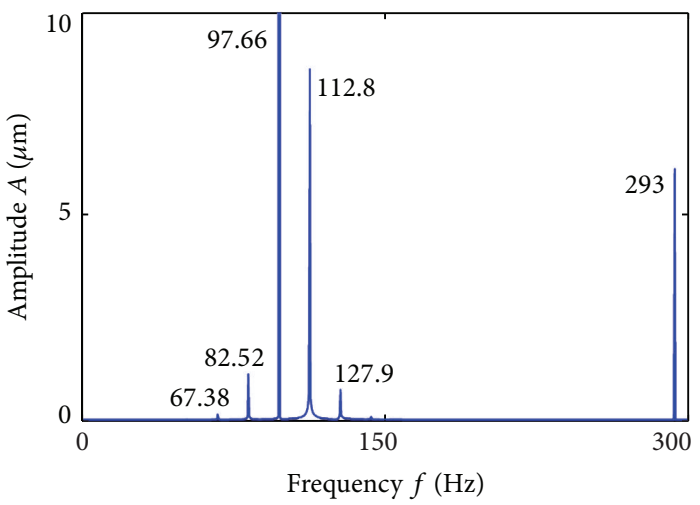

(a3) $7800 \mathrm{r} / \mathrm{min}$ amplitude spectrum

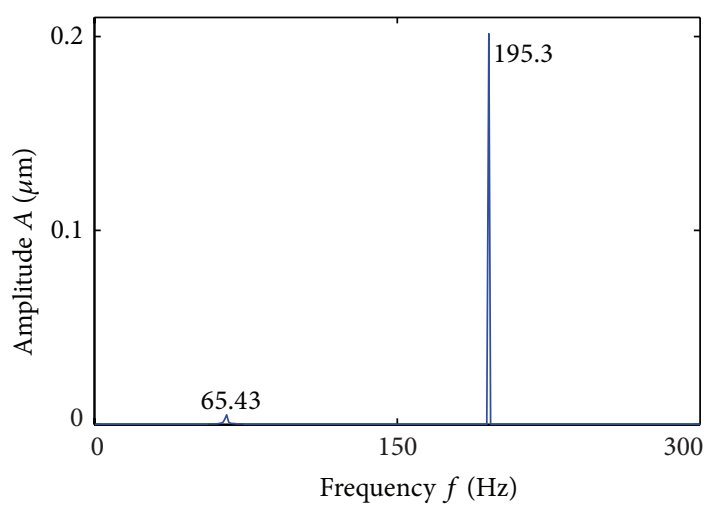

(a4) $12600 \mathrm{r} / \mathrm{min}$ amplitude spectrum

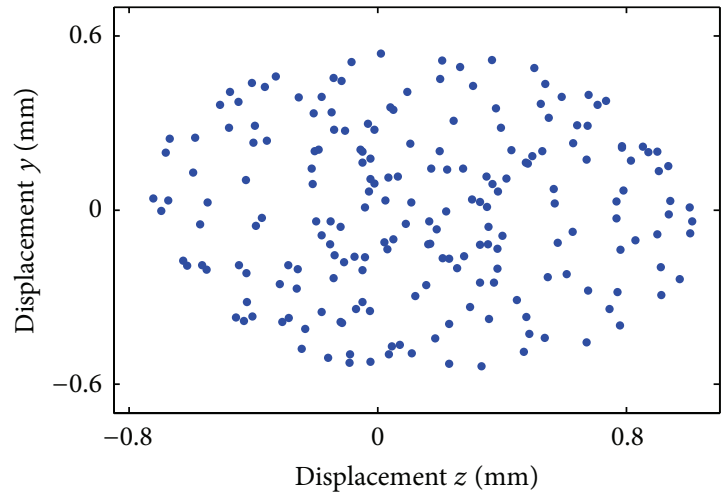

(b1) $1000 \mathrm{r} / \mathrm{min}$ Poincaré

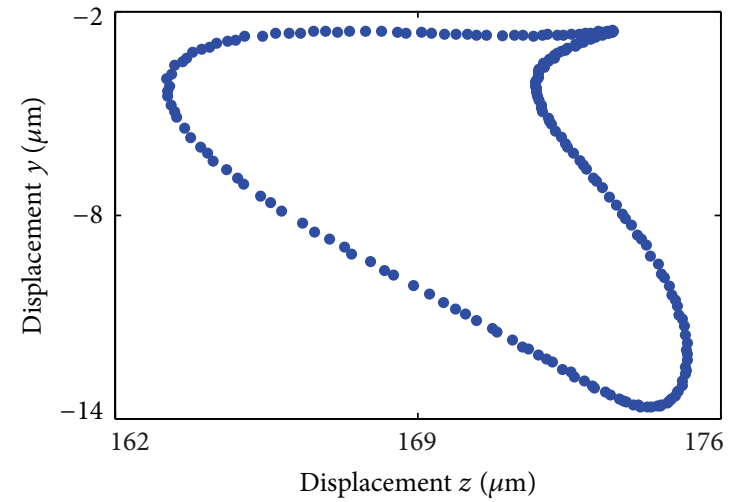

(b2) 2000 r/min Poincaré

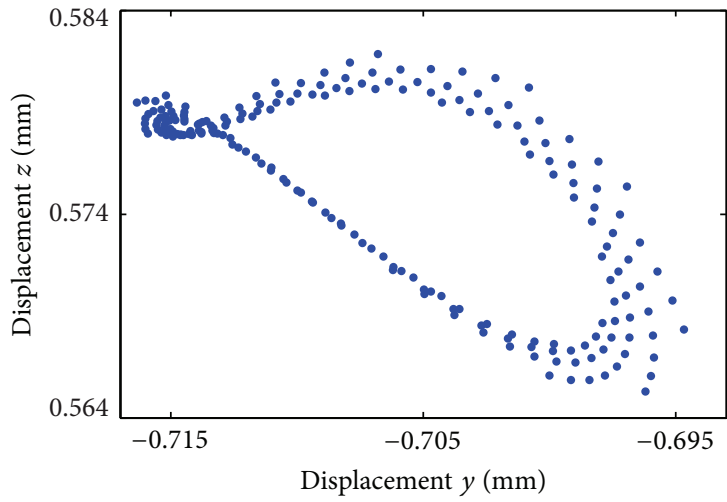

(b3) $7800 \mathrm{r} / \mathrm{min}$ Poincaré

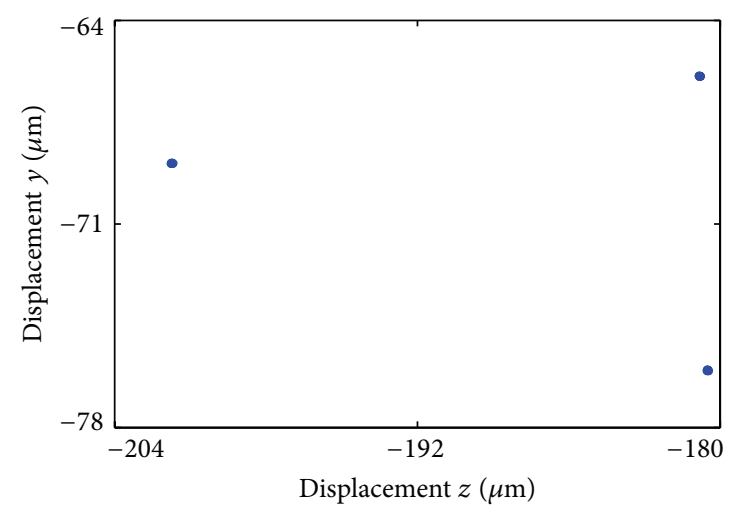

(b4) $12600 \mathrm{r} / \mathrm{min}$ Poincaré

(a)

FIGURE 12: Continued. 


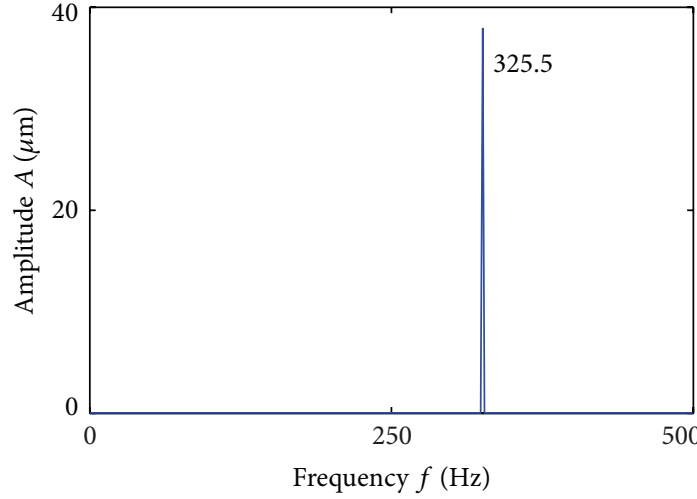

(a5) $20000 \mathrm{r} / \mathrm{min}$ amplitude spectrum

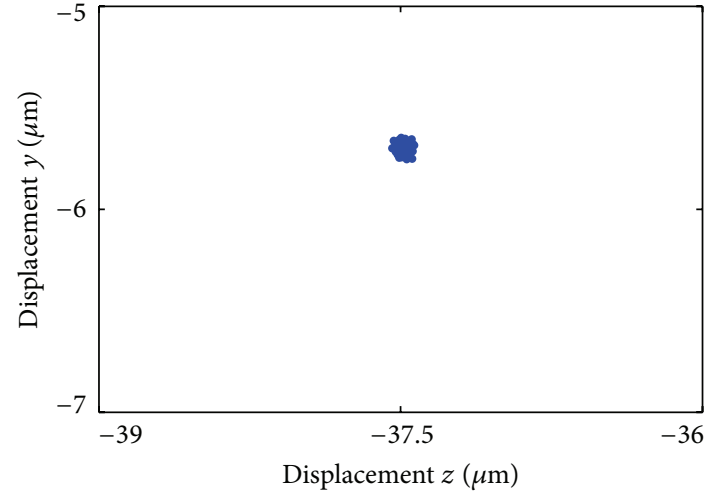

(b5) 20000 r/min Poincaré

(b)

Figure 12: The amplitude diagram and Poincaré mapping of tool point with different speeds.

TABLE 4: The comparison of simulated and experiment results.

\begin{tabular}{lccccc}
\hline & & \multicolumn{2}{c}{ Distribution spring model } & \multicolumn{2}{c}{ Rigid connection model } \\
$\begin{array}{l}\text { Order } \\
i\end{array}$ & Experiment results & Simulated results & Errors & Simulated results & Errors \\
& $A_{i} / \mathrm{Hz}$ & $B_{i} / \mathrm{Hz}$ & $\left|A_{i}-B_{i}\right| / A_{i} \times 100 \%$ & - & - \\
1 & 282 & 276 & $2.13 \%$ & 909 & $3.9 \%$ \\
2 & 875 & 945 & $8 \%$ & 1118 & $6.3 \%$ \\
3 & 1193 & 1139 & $4.53 \%$ & 2157 & $44.9 \%$ \\
5 & 1489 & 1580 & $6.11 \%$ & 2988 & $23.5 \%$ \\
\hline
\end{tabular}

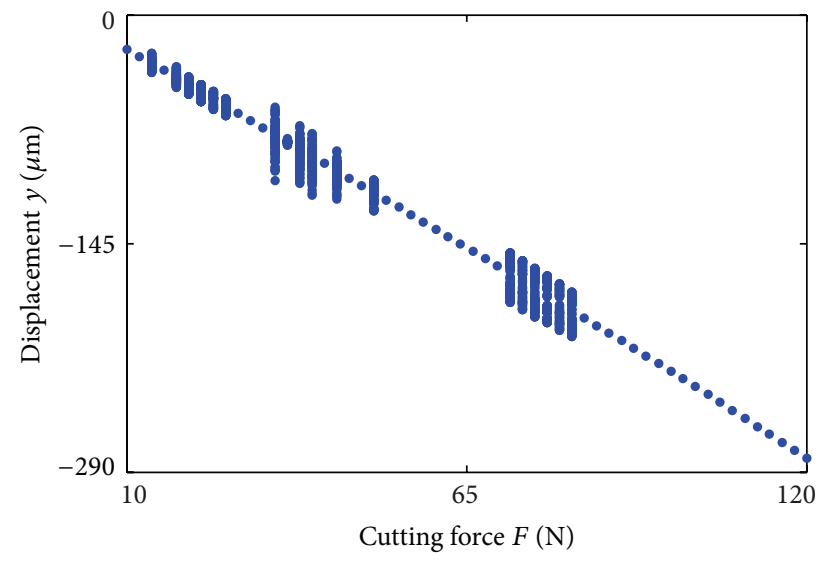

FIGURE 13: Global bifurcation of tool point in $y$ direction with different cutting forces.

is a periodic state. The amplitude spectrum and Poincaré mapping at tool point are shown in Figure 14 to research the stability changing laws with cutting force including $25 \mathrm{~N}$, $40 \mathrm{~N}, 50 \mathrm{~N}, 80 \mathrm{~N}$, and $100 \mathrm{~N}$ clearly.

The amplitude spectrum and Poincaré mapping of tool point at $25 \mathrm{~N}$ are shown in Figures 14(a1)-14(b1). The combination frequencies which existed in the amplitude spectrum included rotating frequency $195.3 \mathrm{~Hz}$. The motion period of tool point is a closed pattern in Poincaré mapping which indicates that the system is in quasiperiodic motion.

The amplitude spectrum and Poincaré mapping of tool point at $40 \mathrm{~N}$ are shown in Figures 14(a2)-14(b2). The continuous spectrum occurs in the amplitude spectrum except the rotating and combination frequency which makes the random points in Poincaré mapping and the stability of tool point chaos.

The amplitude spectrum and Poincaré mapping of tool point at $50 \mathrm{~N}$ are shown in Figures 14(a3)-14(b3). The rotating frequency, a third of rotating frequency, and combination frequencies are in amplitude spectrum together, which leads to the 3 independent closed patterns in the Poincaré mapping. The system is in P-3 quasiperiodic motion state.

The amplitude spectrum and Poincaré mapping of tool point at $80 \mathrm{~N}$ are shown in Figures 14(a4)-14(b4). The state is similar to cutting force in $25 \mathrm{~N}$ which just shows different combination frequencies in amplitude spectrum and the Poincaré mapping is a different shape closed pattern which shows that the system is in quasiperiodic state.

The amplitude spectrum and Poincaré mapping of tool point at $100 \mathrm{~N}$ are shown in Figures 14(a5)-14(b5). There is only the rotating frequency $195.3 \mathrm{~Hz}$ in the amplitude spectrum and one point in Poincaré mapping. The system motion is stable. 


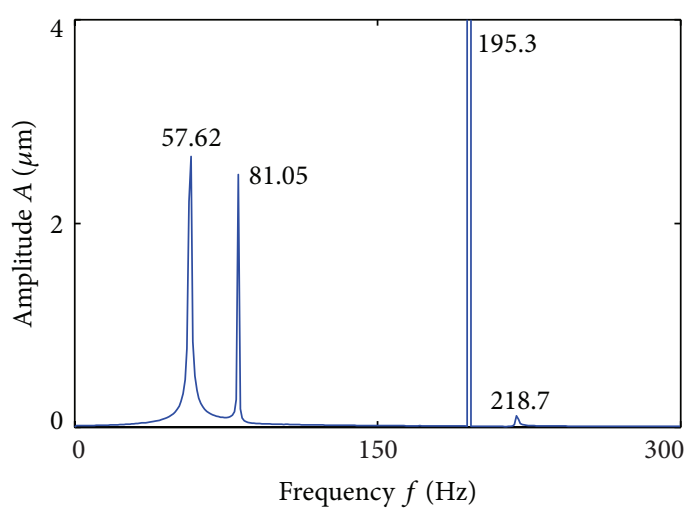

(a1) $25 \mathrm{~N}$ amplitude spectrum

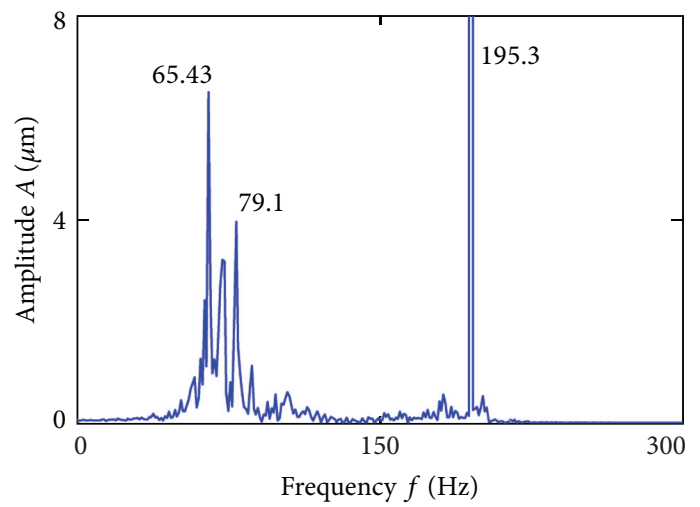

(a2) $40 \mathrm{~N}$ amplitude spectrum

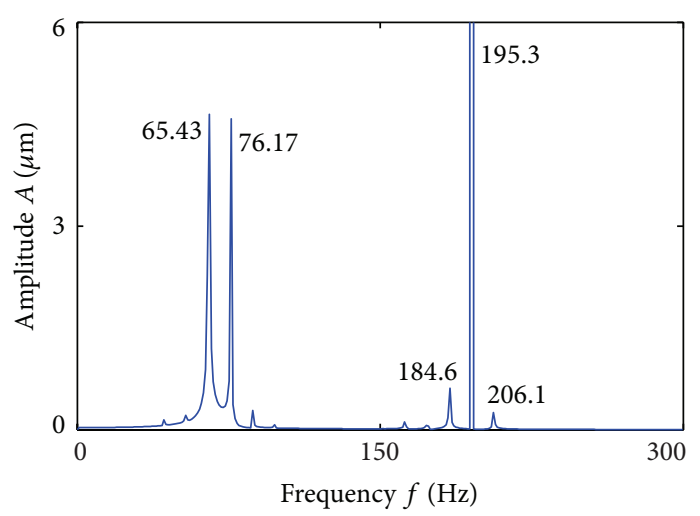

(a3) $50 \mathrm{~N}$ amplitude spectrum

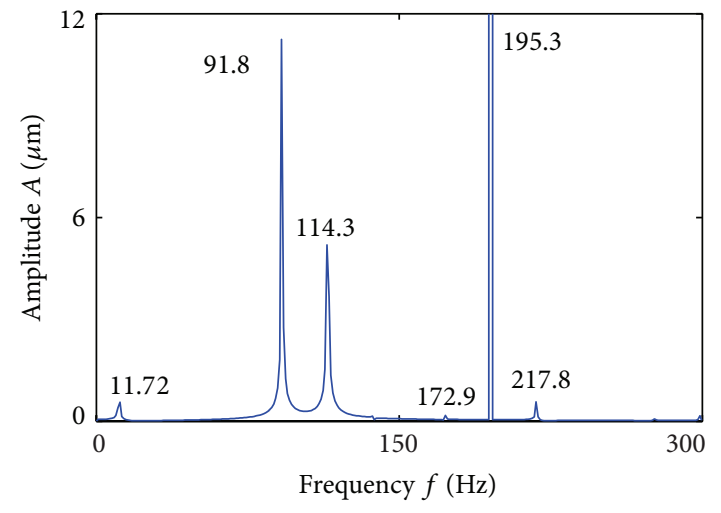

(a4) $80 \mathrm{~N}$ amplitude spectrum

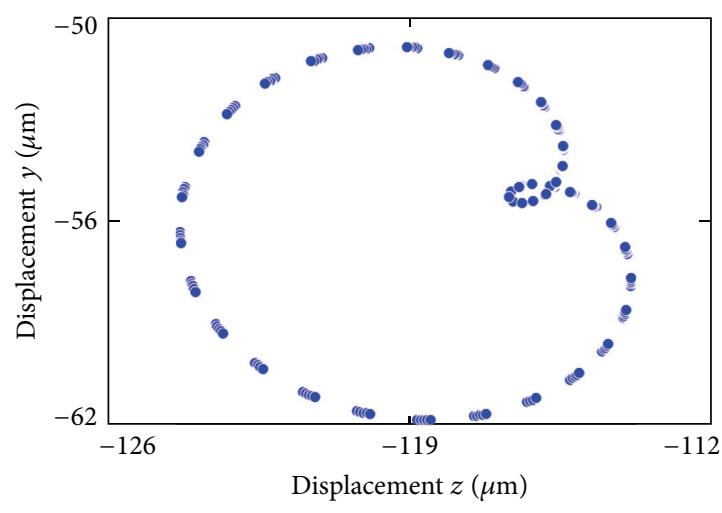

(b1) $25 \mathrm{~N}$ Poincaré

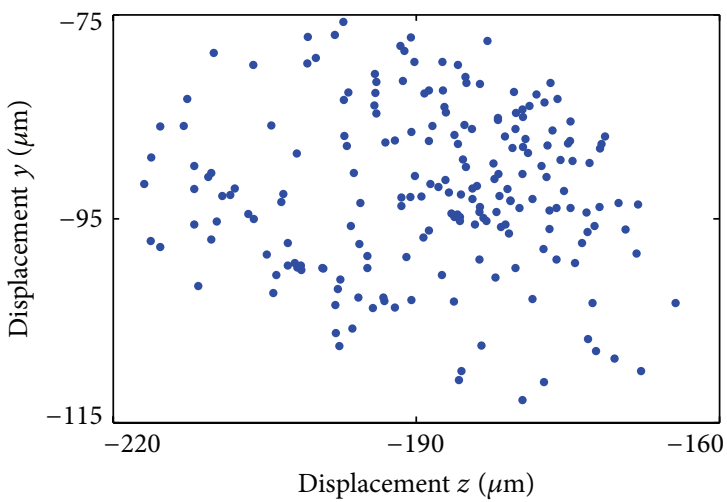

(b2) $40 \mathrm{~N}$ Poincaré

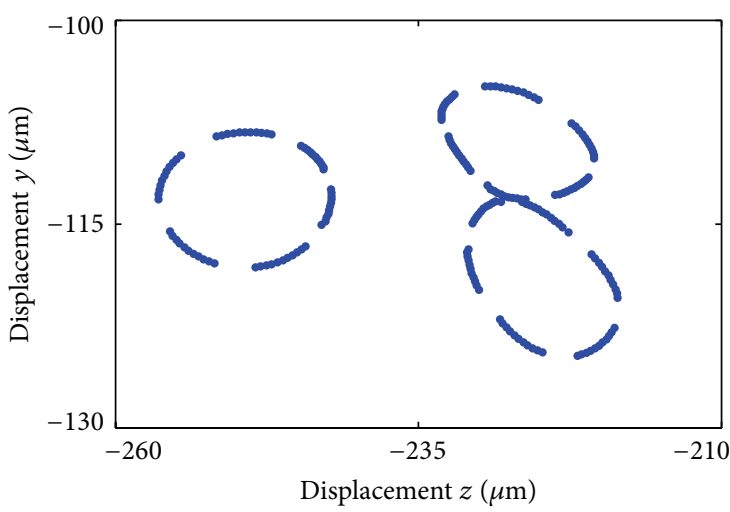

(b3) $50 \mathrm{~N}$ Poincaré

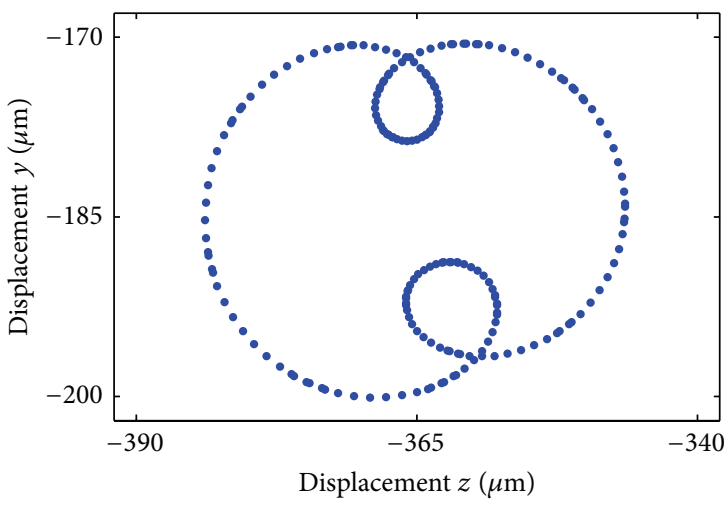

(b4) 80 N Poincaré

(a)

FIgURE 14: Continued. 


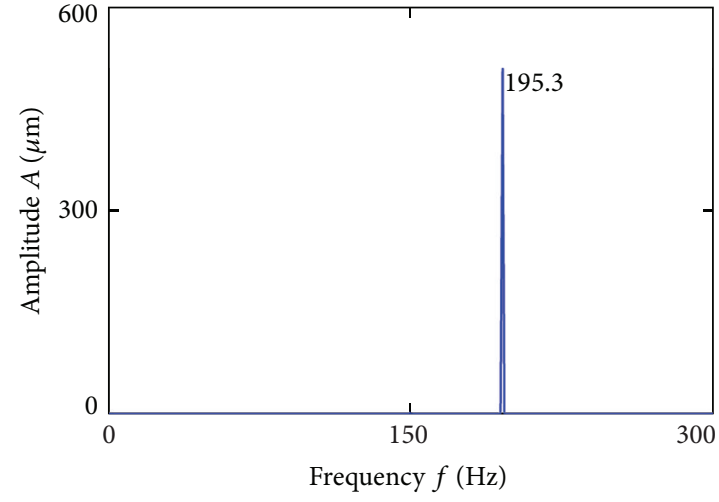

(a5) $100 \mathrm{~N}$ amplitude spectrum

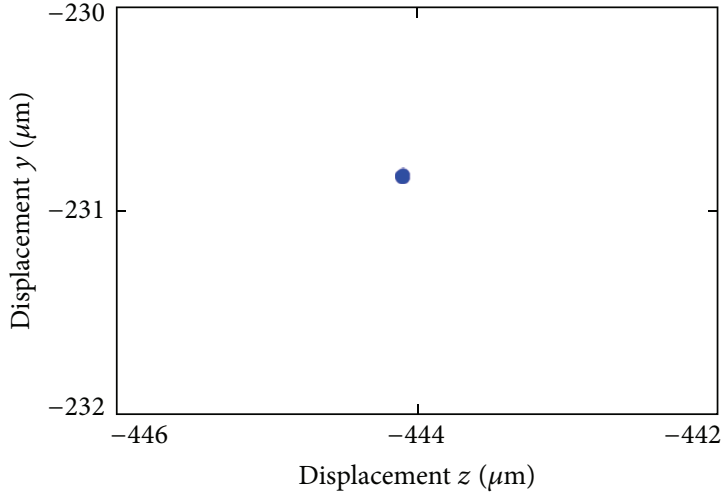

(b5) $100 \mathrm{~N}$ Poincaré

(b)

Figure 14: The amplitude diagram and Poincaré mapping of tool point under different cutting forces.

\section{Conclusions}

In this paper, the dynamic model of high-speed spindle system with spindle-bearing joint and spindle-holder-tool joints has been built. From the practical viewpoint of application, the influence of speed and cutting force has been investigated. Based on the modeling and application results, the following conclusions can be drawn.

(1) A distribution spring model of the spindle-holdertool joints was proposed and applied in the general high-speed spindle FEM model. The rationality of model was verified by comparing the distribution spring connection model, rigid connection model, and the experimental results.

(2) The influence of speed was analyzed. When the speed gradually increases, the nonlinear stability goes through chaos-quasiperiodic transition form between quasiperiodic and chaos-P3 periodicperiodic motions, illustrating that in high speed situation the spindle system is more stable.

(3) The influence of cutting force was analyzed. The spindle system is unstable either in chaos or in quasiperiodic state under low cutting force but more stable under large cutting force. Through the comparison with the actual manufacturing situation, it is found that the dynamic model built in this paper was suitable for large cutting force.

The influence of nonlinear stability on high-speed spindle system includes many other aspects, such as affected parameters of chaotic motion, the stability of thin-walled workpiece, and bearing parameters. How to structure a nonlinear stability evaluation system and to analyze the effects of the parameters may be a good research subject in the future.

\section{Conflict of Interests}

The authors declare that there is no conflict of interests regarding the publication of this paper.

\section{Acknowledgments}

This work was supported by the National Natural Science Foundation of China (50905029), the National Science Foundation for Postdoctoral Scientists of China (2013M541239), the Research Foundation of Education Bureau of Liaoning Province, China (L2013115), and the Fundamental Research Funds for the Central Universities of China (N110603004). National Science Foundation for Postdoctoral Scientists of China (2014M551105), National Basic Research Program of China (2011CB706504).

\section{References}

[1] Y. Cao and Y. Altintas, "A general method for the modeling of spindle-bearing systems," Journal of Mechanical Design, Transactions of the ASME, vol. 126, no. 6, pp. 1089-1104, 2004.

[2] Y. C. Shin, "Bearing nonlinearity and stability analysis in high speed machining," Journal of engineering for industry, vol. 114, no. 1, pp. 23-30, 1992.

[3] G. Chen, C. G. Li, and D. Y. Wang, "Nonlinear dynamic analysis and experiment verification of rotor-ball bearings-supportstator coupling system for aeroengine with rubbing coupling faults," Journal of Engineering for Gas Turbines and Power, vol. 132, no. 2, Article ID 022501, pp. 1-9, 2010.

[4] E. I. Rivin, "Tooling structure: interface between cutting edge and machine tool," Annals of the ClRP, vol. 49, no. 2, pp. 591609, 2000.

[5] T. L. Schmitz, M. A. Davies, and M. D. Kennedy, “Tool point frequency response prediction for high-speed machining by RCSA," Journal of Manufacturing Science and Engineering, Transactions of the ASME, vol. 123, no. 4, pp. 700-707, 2001.

[6] M. Namazi, Y. Altintas, T. Abe, and N. Rajapakse, "Modeling and identification of tool holder-spindle interface dynamics," 
International Journal of Machine Tools and Manufacture, vol. 47, no. 9, pp. 1333-1341, 2007.

[7] A. Ertürk, H. N. Özgüven, and E. Budak, "Effect analysis of bearing and interface dynamics on tool point FRF for chatter stability in machine tools by using a new analytical model for spindle-tool assemblies," International Journal of Machine Tools and Manufacture, vol. 47, no. 1, pp. 23-32, 2007.

[8] H. Yumei, F. Weiping, C. Lixin et al., "The research of dynamic characteristic parameters on normal joints," Chinese Journal of Mechanical Engineering, vol. 29, no. 3, pp. 74-78, 1993.

[9] S. Zhang, X. Ai, and J. Zhao, "FEM-based parametric optimum design of spindle/toolholder interfaces under high rotational speed," Chinese Journal of Mechanical Engineering, vol. 40, no. 2, pp. 83-91, 2004.

[10] W. Winfough, Issues of dynamics in high-speed milling of aluminium aircraft structures [Ph.D. thesis], University of Florida, Gainesville, Fla, USA, 1995.

[11] F. Koenigsberger and J. Tlusty, Machine Tool Structures-Vol. I: Stability Against Chatter, Pergamon, New York, NY, USA, 1967.

[12] S. A. Tobias, Machine Tool Vibration, Blackie, London, UK, 1965.

[13] H. E. Merrit, "Theory of self-excited machine tool chatter," Journal of Manufacturing Science and Engineering, vol. 87, no. 4, pp. 447-454, 1965.

[14] S. S. Park, Y. Altintas, and M. Movahhedy, "Receptance coupling for end mills," International Journal of Machine Tools and Manufacture, vol. 43, no. 9, pp. 889-896, 2003.

[15] Y. Altintaş and E. Budak, "Analytical prediction of stability lobes in milling," Annals of the ClRP, vol. 44, no. 1, pp. 357-362, 1995.

[16] B. P. Mann, T. Insperger, P. V. Bayly, and G. Stépán, "Stability of up-milling and down-milling," International Journal of Machine Tools and Manufacture, vol. 43, no. 1, pp. 25-40, 2003.

[17] T. L. Schmitz, J. C. Ziegert, and C. Stanislaus, "A method for predicting chatter stability for systems with speed-dependent spindle dynamics," in Proceedings of the 32th North American Manufacturing Research Conference (NAMRC '04), pp. 17-24, June 2004.

[18] T. Weixiao, Research on High-Speed Machining Stability and Dynamic Optimization, Shandong University, Jinan, China, 2005.

[19] A. Tang and Z. Liu, "Modeling and simulation of threedimensional stability lobes of milling thin-walled plate," in Proceedings of the IEEE International Conference on Automationand Logistics (ICAL '08), pp. 532-535, September 2008.

[20] F. Abrari, M. A. Elbestawi, and A. D. Spence, "On the dynamics of ball end milling: modeling of cutting forces and stability analysis," International Journal of Machine Tools and Manufacture, vol. 38, no. 3, pp. 215-237, 1998.

[21] C. H. Dai Depai, "The nonlinear theoretic research about cutting chatter of mchine tool," Journal of Vibration Engineering, vol. 5, no. 4, pp. 335-342, 1992.

[22] S. Qinghua, High-Speed Milling Stability and Machining Accuracy, Shandong University, Jinan, China, 2009. 

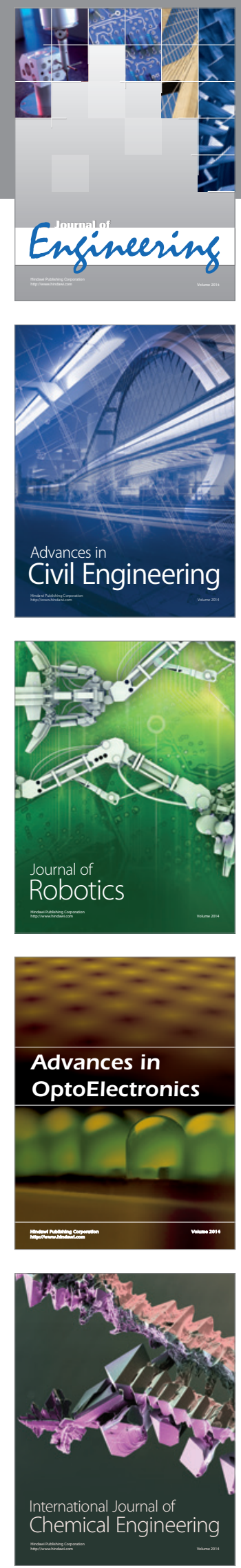

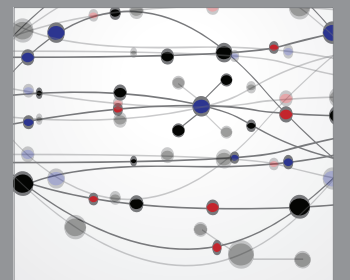

The Scientific World Journal
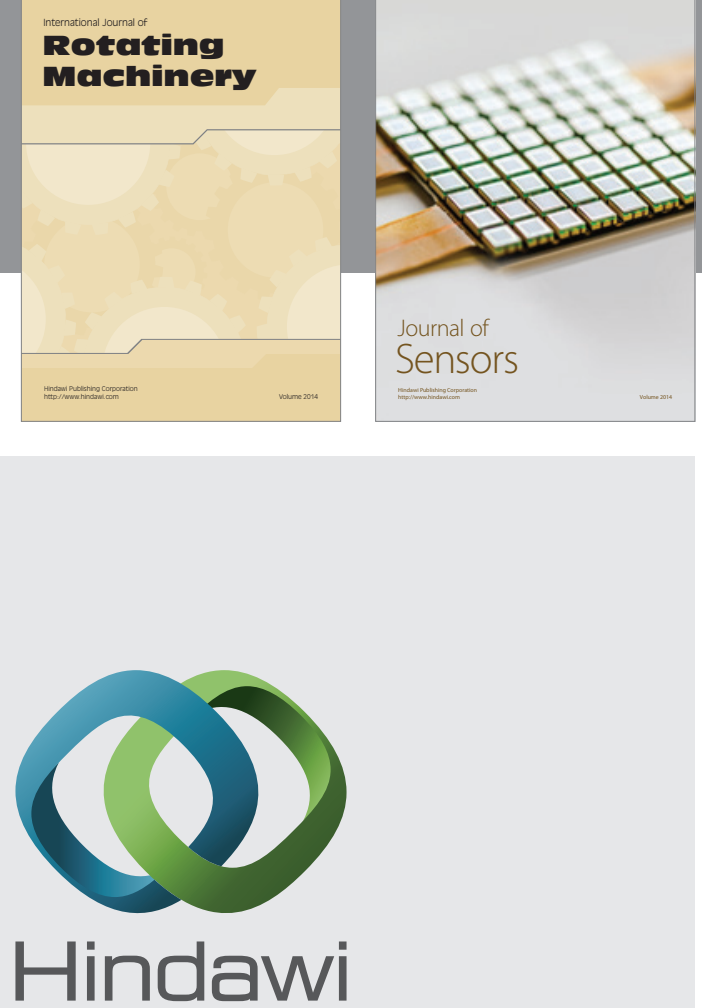

Submit your manuscripts at http://www.hindawi.com
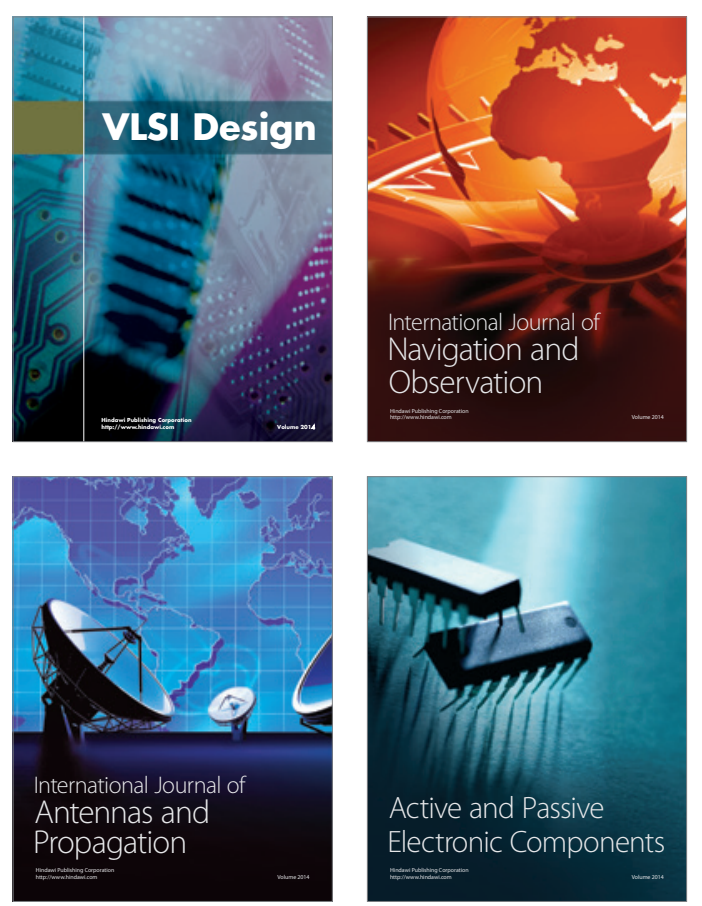
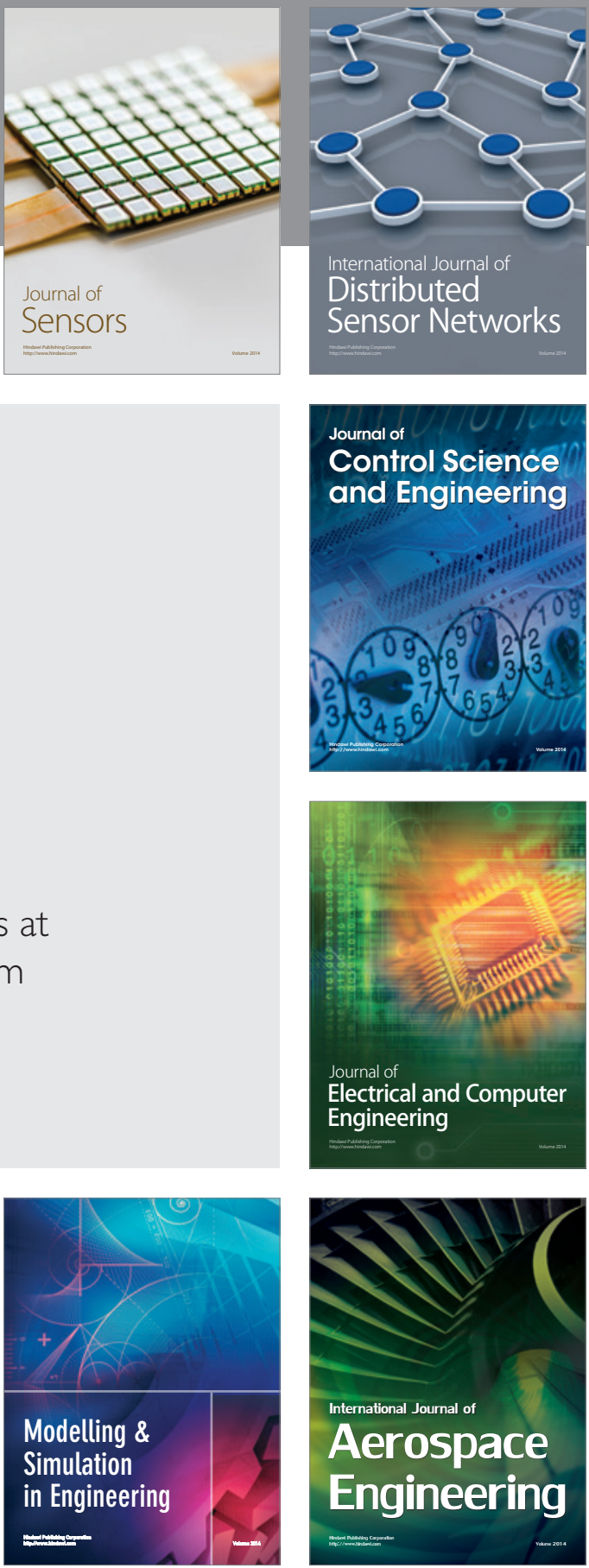

Journal of

Control Science

and Engineering
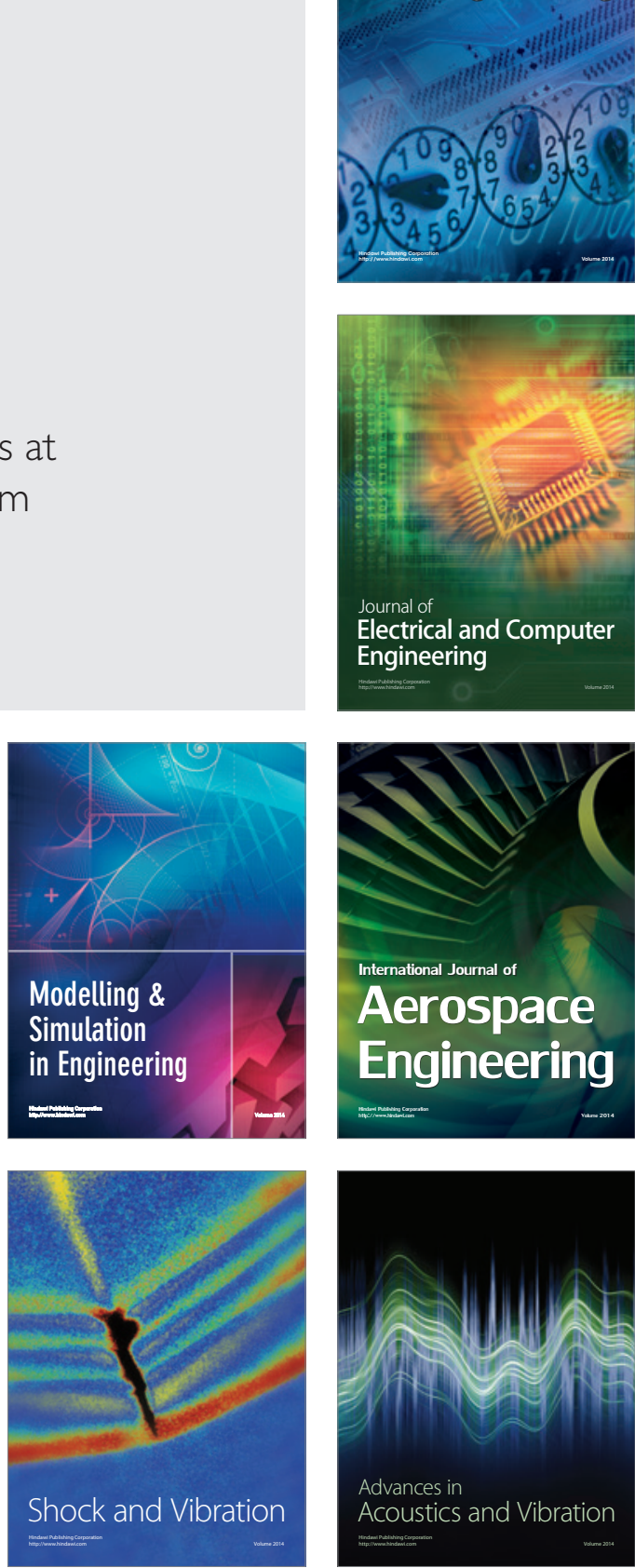